\title{
Comer bien, comer mal: la medicalización del comportamiento alimentario
}

\author{
Mabel Gracia-Arnaiz, Dra en Antrop Soc.(1)
}

\section{Gracia-Arnaiz M. \\ Comer bien, comer mal: la medicalización del comportamiento alimentario. Salud Publica Mex 2007;49:236-242.}

\begin{abstract}
Resumen
En este artículo se analiza el progresivo proceso de medicalización del comportamiento alimentario por el cual una buena parte de los motivos pragmáticos o simbólicos que articulan la selección y el consumo humano de alimentos se ha sustituido por otros de orden nutricional. La normalización dietética se ha concretado, en particular en los contextos de relativa abundancia de alimentos, en torno de la dieta equilibrada, un patrón alimentario basado en la restricción y promoción del consumo de ciertos alimentos y nutrientes -qué y cuánto comer-y en la prescripción de un conjunto de pautas relativas a cómo, cuándo y con quién hacerlo, y cuyos objetivos han sido no sólo regular los cuidados del cuerpo y la salud, sino promover, a su vez, un cierto orden social y ético.
\end{abstract}

Palabras clave: alimentación; cultura; medicalización; normalidad dietética; obesidad

\author{
Gracia-Arnaiz M. \\ Eating well, eating poorly and the medicalization \\ of eating behavior. \\ Salud Publica Mex 2007;49:236-242.
}

\section{Abstract}

This article describes how the progressive process of medicalization of food is inscribed within a biomedical logic that attempts to substitute many of the economic, adaptative and symbolic reasons that have ethnographically and historically conditioned eating habits in all cultures, exclusively for reasons of a dietetic nature.According to this logic, most of the population of modern societies does not know how to eat because they are victims of or have been made ill by the current profusion of food. They must, therefore, learn how to eat well, which in terms of eating practices means eating a little of everything, in moderation and in a balanced way. The main aim of current nutritional education is to (re)establish a supposed dietetic normality as far as food consumed, quantities, number of intakes and meal structures are concerned.

Key words: food; culture; medicalization; dietetic normality; obesity
$\mathrm{L}^{2}$ a finalidad de este artículo es profundizar en los - motivos por los cuales una buena parte de las razones pragmáticas y simbólicas que articulan la selección y el consumo alimentario tratan de reemplazarse por otras de carácter nutricional y, a partir de ahí, trazar una reflexión general sobre el proceso de medicalización de la alimentación contemporánea. Con este objetivo, se vinculan las propuestas de Mennell y Turner acerca de la civilización del apetito y la afinidad entre la gestión facultativa de la dieta y la expansión del capitalismo. Esta propuesta tomó como referente lo que aquí se define como el proceso de normalización dietética, esto es, la construcción de un patrón alimentario específico -la dieta equilibrada-con base en la restricción o promoción del consumo de ciertos alimentos y la prescripción de un conjunto de disposiciones relacionadas con el modo

(I) Departamento de Antropología, Filosofía y Trabajo Social, Universidad Rovira i Virgili.Tarragona, España.

Fecha de recibido: 18 de septiembre de 2006 - Fecha de aceptado: 2 I de marzo de 2007 Solicitud de sobretiros: Dra. Mabel Gracia Arnaiz. Departamento de Antropología, Filosofía y Trabajo Social, Universidad Rovira i Virgili. 43005 Tarragona, España. Correo electrónico: mabel.gracia@urv.cat 
de establecerlo, cuándo y en compañía de quiénes; sus objetivos son homogenizar la vida cotidiana. En las páginas que siguen se muestran, a través de la problematización de la obesidad, algunas de las limitaciones de un marco interpretativo que analiza el comportamiento alimentario, en particular desde la óptica de la enfermedad y salud, sin tomar en cuenta, para ello, la compleja naturaleza de la alimentación y la cultura.

\section{Comer mucho, comer poco, comer nada}

Los usos y las actitudes hacia los alimentos son tantos como variados y se manifiestan al expresar el significado de comer, su utilidad o la razón de hacerlo. ${ }^{1}$ En ocasiones es posible comer en abundancia como forma para medir las propias capacidades o responder a los apremios que la cultura representa. En la sociedad kwakiutl de la costa noroeste americana, la acumulación de abundante comida y su posterior distribución entre el conjunto de la comunidad durante la celebración del potlatch constituían un comportamiento que debía acompañar sin excepción a toda pretensión de convertirse en grandes hombres, ${ }^{2}$ del mismo modo, en la época medieval, nobles y terratenientes ingleses podían sentarse a la mesa para consumir festines consistentes en 20 o 30 platos distintos de carnes de diversos tipos con el propósito sociopolítico de simbolizar el poder ejercido sobre el pueblo llano. ${ }^{3}$ Por su parte, entre los banyankole, un pueblo pastor del este de África, cuando se prepara a una mujer para el matrimonio, a la edad de ocho años, ya no se le permite jugar ni correr, sino que debe permanecer en casa y beber grandes cantidades de leche diarias hasta engordar de tal modo que, al cabo de un año, apenas si puede andar con pasos vacilantes. Las mujeres de la corte, la madre del rey y sus esposas, son las más gruesas y ello no sólo es signo de estatus sino de belleza. ${ }^{4}$

Estos ejemplos muestran que las prácticas alimentarias no responden tan sólo a la necesidad biológica de llenar el cuerpo de combustible, según propondría una visión mecanicista del organismo humano, ${ }^{5}$ sino a las condiciones materiales y las representaciones simbólicas que articulan las relaciones sociales en las diferentes sociedades. Observar que el alimento no es sólo algo para nutrir no significa soslayar que también es una sustancia para subsistir y que, cuando escasea durante periodos prolongados, se corre el peligro de morir de hambre. ${ }^{6,7}$ Sin embargo, como señala Mintz, hambre hay de muchos tipos. Ayunar porque no hay nada o poco que comer, como le sucede a millones de personas hoy en día, ${ }^{9}$ es el modo más dramático de conocer el poder de la comida. No obstante, en ocasiones se deja de comer aun si hay alimentos disponibles. Del mismo modo que el hartazgo, el autoayuno ha constituido una respuesta cultural a situaciones concretas en contextos determinados. ${ }^{10}$ Ha sido una actitud de sufragistas, huelguistas, artistas del hambre o ascetas. En consecuencia, parece importante precisar el significado de estas prácticas alimentarias: ¿poder, resistencia, reivindicación, solidaridad, purificación, sumisión?

Es difícil creer en la voluntariedad de estos actos, al menos en un sentido arbitrario y, en realidad, el dominio de las necesidades fisiológicas, sobre todo las sexuales y las alimentarias, ha sido motivo de reconocimiento social en numerosas culturas. ${ }^{11}$ Por ejemplo, el ayuno por motivos religiosos, que acataron las mujeres canonizadas por el clero masculino durante la Edad Media, era una práctica razonable dentro de una lógica ascética y una ideología que sublimaba el rechazo de lo terrestre como medio eficaz de acercarse a Dios. ${ }^{12}$ Se sabe que estas mujeres ayunaron y que algunas murieron por inanición, pero se ignoran otras posibles razones de su reclusión monacal. ¿Controlaron el apetito sólo para demostrar piedad, abnegación, amor a Dios o arrepentimiento? Ésta es la respuesta más esgrimida. Sin embargo, también se ha señalado que no comer fue una forma de resistencia vinculada con el comportamiento de jóvenes que prefirieron rechazar las imposiciones familiares respecto de las expectativas matrimoniales y que destinaron su vida a la oración y el sacrificio. $\mathrm{Al}$ fin y al cabo, entregarse a Dios, o casarse con él, podía ser más gratificante que hacerlo con un ser humano. ${ }^{13}$ Por otro lado, la Iglesia Católica dictó numerosos ayunos en función de las fluctuaciones provocadas por los ciclos agrícolas y la distribución desigual de los alimentos. ${ }^{14}$ De esta forma, la abstinencia sirvió para aceptar religiosamente las carencias intrínsecas de una sociedad no abundante y socialmente muy desigual, de tal manera que la necesidad fue virtud y la gula, el consumo excesivo de comida y bebida, pecado capital.

Si bien es cierto que las formas de ayuno voluntario alcanzan mayor relevancia en las sociedades industrializadas, sea porque hay comida en abundancia o porque en una situación novedosa de profusión alimentaria parece difícil, y para algunos absurdo, limitar o negar una necesidad tan primaria como alimentarse, conviene preguntarse, del mismo modo, qué lógica subyace hoy a estas prácticas y relativizar el carácter anómalo o patológico atribuido en estos contextos a comer mucho, poco o nada.

\section{La construcción social de la normalidad dietética}

Si se toman en cuenta los ejemplos expuestos aquí, podría sorprender el hecho de que en la actualidad los 
comportamientos alimentarios los refieran las autoridades políticas y los expertos sanitarios, sobre todo a través del discurso de la salud o la enfermedad, y que se tornen objeto de incumbencia, en primera instancia, de orden médico. Sin embargo, no hay razones para tal sorpresa. La larga tradición de la medicina occidental de proveer información y consejos sobre la cantidad y la composición de la comida sana, la regulación del peso y la prevención de enfermedades ha contribuido a privilegiar las funciones biológicas de los alimentos a lo largo del tiempo y, dada su influencia en otros sistemas médicos, también del espacio. Que la interpretación biomédica enfatice los aspectos fisiológicos en detrimento de los sociales responde a una lógica que se ha construido al compás de la medicalización de la alimentación. ${ }^{15}$ Los orígenes de esto se remontan a los tratados hipocráticos que, al aludir al cuidado del cuerpo y la salud, hicieron célebre en la Antigüedad clásica la máxima: que tus alimentos sean tu medicina. ${ }^{16}$ En adelante, la aplicación de las normas dietéticas pretendió sustituir una buena parte de los motivos pragmáticos o simbólicos sobre los cuales se articulan la selección y el consumo alimentario por otros de interés médico. ${ }^{17}$ Por influencia de la teoría mecanicista, esta tendencia se acentuó durante los siglos XVII y XVIII al conceder más atención a las cualidades sanitarias de los alimentos. ${ }^{18} \mathrm{El}$ conocido físico inglés George Cheyne ${ }^{19}$ asumió la metáfora mecánica del cuerpo-instrumento formado de circuitos y flujos- para explicar que la comida constituía el combustible que abastecía la máquina humana y afirmar que la dieta rica, es decir, la consumida opíparamente por las elites constituía el origen de numerosas enfermedades y, por tanto, había que modificarla.

El incremento de la producción coincidió en los países occidentales con la distribución menos desigual de los alimentos; en el siglo XIX la prescripción de límites al consumo excesivo se generalizó a través de sucesivas reformas alimentarias. ${ }^{20}$ Durante este período se manifestó con fuerza lo que Mennell ${ }^{21}$ ha denominado el proceso de civilización del apetito con base en la propuesta de Elías. ${ }^{22}$ Dicho proceso, producido a lo largo de varios siglos, supuso la modificación de las pautas del comportamiento siempre, según este autor, en una dirección determinada: la civilización se plasmó en un cambio progresivo del ejercicio de constreñimientos principalmente externos (ecológicos, económicos, simbólicos) hacia el desarrollo de constreñimientos internos, los cuales ejercieron las personas sobre sí mismas (dietas, higiene). Por ejemplo, en las áreas de influencia protestante, y en general cristiana, las recomendaciones dietéticas pasaron a formar parte de un reclamo ético. Según éstas, el cuidado del cuerpo era una responsabi- lidad moral y se estableció, de acuerdo con Turner, ${ }^{23,24}$ una afinidad paralela entre la gestión facultativa de la dieta y la expansión del capitalismo: la restricción evitaba la gula entre las élites y una nutrición suficiente mantenía a los trabajadores como mano de obra activa. En Estados Unidos, estos principios los difundieron en los hogares de las clases obreras los representantes de la New Nutrition. Éstos, un grupo de nutricionistas, reformadores sociales y especialistas en economía doméstica, pretendieron organizar el gasto alimentario al cambiar, sin mucho éxito, las costumbres culinarias. ${ }^{25}$

En la actualidad, la normalización dietética se ha concretado en torno a la dieta equilibrada, esto es, un patrón alimentario basado en la restricción o promoción del consumo de ciertos alimentos* (qué y cuánto comer) y la prescripción de un conjunto de pautas relativas a cómo, cuándo y con quién hacerlo, cuyos objetivos son lograr una nutrición sana exenta de riesgo para la salud. ${ }^{27}$ No obstante, dichos objetivos implican normalizar la vida cotidiana. ${ }^{26}$ En efecto, las múltiples guías publicadas para la promoción de una alimentación saludable, ${ }^{27-29}$ al pretender enseñar a comer bien, es decir, en compañía, ni de prisa ni despacio, a masticar pausadamente, entre tres y cinco veces al día, en horarios bien definidos y en variedad y cantidades justas, promueven una regulación de los modos de vida basada, a su vez, en una responsabilidad del cuidado de sí mismo y la exigencia de una verdadera competencia dietética. ${ }^{30}$ Estas cuestiones se reconocen a través de la medicalización de la obesidad en las sociedades occidentales.

En la progresiva, que no reciente, problematización de la gordura ${ }^{31}$ han intervenido criterios culturales vinculados con el menosprecio de la glotonería o el valor de la delgadez corporal y, de forma más reciente, criterios sanitarios y económicos por su efecto negativo en la salud y los costos asistenciales. ${ }^{\ddagger} \mathrm{Al}$ definirla como una acumulación excesiva o anormal de grasa, ${ }^{33}$ la obesidad se considera, dado el incremento de su prevalencia en todo el mundo, una enfermedad de carácter plurifactorial y epidémico ${ }^{34}$ que puede prevenirse con estilos de vida saludables que procuren el equilibrio entre las

\footnotetext{
* Las recomendaciones alimentarias relacionadas con esta dieta pueden consultarse en http:// www.msc.es/ ciudadanos/ proteccionSalud/infancia/alimentacion/tema2.htm.

* Según la OMS, más de 1600 millones de personas tienen sobrepeso y, de ellas, al menos 400 millones son obesas (http: / / www.who.int). En España, 14.5\% de la población adulta es obesa y el sobrepeso asciende a $38.5 \%$, mientras que los costos directos e indirectos secundarios a esta enfermedad suponen $7 \%$ del gasto sanitario total, es decir, alrededor de 2500 millones de euros anuales. ${ }^{32}$
} 
calorías ingeridas y las calorías gastadas. ${ }^{32}$ Como si comer, de manera absoluta, se tratara de una simple operación matemática. Desde que se vinculó con el incremento de la morbilidad y mortalidad debidas a enfermedades crónicas no transmisibles -hipertensión, diabetes, infartos-y el establecimiento del índice de masa corporal (IMC), ${ }^{*}$ no sin polémica, ${ }^{35,36}$ como el indicador más utilizado para definirla, se instruyó a toda la población -al margen de su heterogeneidad biosocial- para que procurara mantenerse en los límites de su IMC mediante la vigilancia estricta del peso, el seguimiento de la dieta equilibrada y la práctica de ejercicio físico. En general, se trata de comer menos y moverse más ${ }^{37}$ porque en la actualidad estar gordo se ha convertido, en particular en los países industrializados, en sinónimo de estar enfermo.

Según este enfoque medicalizado de la gordura, la obesidad y el sobrepeso, se asimila como evidente, sin serlo aún, que la grasa mata, que la obesidad es en sí patológica y que todos los obesos son sin excepción enfermos o lo serán. ${ }^{38}$ La obesidad representa un signo de trasgresión normativa y la consecuencia de aquello que no debe hacerse: comer mucho y ser ocioso. La percepción negativa de la gordura entre los profesionales de la salud se ha construido, a menudo, sobre la idea de que una vez instaurada, es una enfermedad intratable por la tendencia a ganar peso y por la supuesta falta de interés o voluntad de la persona obesa a hacer dieta y ejercicio para perderlo. ${ }^{39}$ Aunque en el ámbito sanitario hay nutricionistas que intentan tratar al paciente como un ser global al adaptar sus consejos a su particularidad biopsicosocial, ${ }^{40}$ son muy frecuentes las recriminaciones relativas a las conductas alimentarias en razón de sus dimensiones morales; esto hace sentir a los sujetos faltos de criterio -o de competencias- e irresponsables: con frecuencia... cuando no nos sentimos bien lo compensamos comiendo y bebiendo, aunque no tengamos apetito y en contra de nuestro raciocinio y nuestra salud. ${ }^{32} \mathrm{Al}$ prescribir dietas de adelgazamiento, numerosos facultativos consideran que los portadores de la anomalía (el exceso de grasa) son responsables de su disfunción:41,42 si usted está obeso es porque no sabe o no quiere comer bien. En el caso de que las personas gordas sean niños, tal responsabilidad se traslada con facilidad a los progenitores, como ha sucedido en fecha reciente en Gran Bretaña, cuando la madre de Connor McCreaddie, un niño de nueve años y 89 kilos de peso, estuvo a punto de perder la custodia de su hijo acusada de "negligencia". ${ }^{43}$

\footnotetext{
* El IMC es igual a la masa en kilogramos dividida por el cuadrado de la estatura en metros.
}

\section{"Comer bien", “comer mal”: ¿una cuestión sólo de educación?}

Concebir los modos de vida como desordenados o inadecuados a consecuencia de una civilización abundante $-y$ decadente- ${ }^{44}$ ha servido a los expertos sanitarios no sólo para legitimar las acciones de cambio en una dirección determinada -civilizar el apetito- sino para reproducir y mantener sus prácticas a través de la prevención. El argumento defendido para la educación nutricional en el caso de la obesidad es triple: ${ }^{45}$ es posible vivir mejor si se observa una dieta equilibrada, se trabaja más si se goza de una buena salud y representa menos costos para la colectividad. Los objetivos sanitarios, económicos y morales de tales propuestas son, por tanto, claros.

Ahora bien, conviene formularse algunas cuestiones respecto de estos planteamientos y tratar de dilucidarlas. En primer lugar, ¿es cierto que no se sabe comer?, ¿es verdad que los comportamientos alimentarios están más desestructurados que en épocas anteriores y que dicha desestructuración afecta a todos por igual? Si es así sorprende, de nueva cuenta, que en estas poblaciones aumentara la esperanza de vida de forma vertiginosa y ello se atribuya, en buena medida, a la mejoría del estado nutricional de determinados grupos sociales. ¿No es entonces que la interpretación biomédica de este no-saber es consecuencia, cuando menos, de su interés por influir en todos los ámbitos de la vida cotidiana?

Numerosas recomendaciones nutricionales han alterado los comportamientos alimentarios en favor de mejorar la salud colectiva al objetar, con mayor o menor acierto, la bondad de los hábitos anteriores y proponer otros nuevos. Ha sido el caso, por ejemplo, de las contradictorias prescripciones médicas sobre la lactancia infantil a lo largo del siglo pasado en España y que en su momento desautorizaron de manera arbitraria las competencias maternas. ${ }^{46}$ Lo que se considera bueno o malo, normal o patológico en salud se transforma a través del tiempo y el espacio y por esta razón conviene ser prudente al establecer dogmas de base científica. ${ }^{47}$ Hoy día, en un contexto muy medicalizado, cada vez más prácticas alimentarias se consideran de riesgo (malas) porque los saberes expertos las han objetivado y cuantificado. ${ }^{48}$ Por sí mismo, el "riesgo" es un concepto que aparece en las sociedades modernas cuando, en concordancia con el desarrollo del cálculo estadístico, se cree que ante la amenaza de un daño -físico o psíquico- algo puede y debe hacerse para evitarlo. ${ }^{49}$

Considerar que evitar las conductas arriesgadas depende, en gran medida, del individuo supone no comprender, tal como argumentan Douglas y Wildas$\mathrm{ky}^{50}$ y Boltanski y Thévenot, ${ }^{51}$ que los sujetos perciben o representan un objeto $\mathrm{u}$ actividad como arriesgada, 
según sean el entorno económico y político, el sistema de valores y creencias y la posición que, por razones de género, clase, edad o grupo étnico, ocupan en la estructura social de una sociedad particular. Y son estos condicionantes estructurales los que, organizados en sistemas complejos, afectan a las personas y determinan si para ellas, al final, un comportamiento es preferible o evitable respecto de otros. Los hábitos alimentarios no están determinados de modo exclusivo por la preocupación por la salud o la enfermedad. Para algunos individuos el riesgo de engordar no consiste en contraer una obesidad mórbida, sino en dejar de tener un cuerpo socialmente aceptable $^{52}$ y, pese a ello, otras personas gordas no se inquietan por su sobrepeso, sino que hacen de éste una razón para exigir el reconocimiento institucional de su particularidad..$^{53}$

$\mathrm{Al}$ atender la diversidad de significados atribuidos a la obesidad y, por otro lado, las diferentes causas y efectos de ésta en la salud de las poblaciones, no parece sensato considerarlas aún como una consecuencia de asumir irresponsablemente conductas de riesgo. ${ }^{54}$ En la reciente medida diseñada en España para hacer frente a la obesidad, ésta se define como un mal de carácter ambiental que hay que abordar sin recurrir a una campaña represiva, interpretada por el ciudadano como prescriptiva o de prohibición, ${ }^{32}$ sin soslayar que los factores ambientales o del entorno juegan un papel primordial en el desarrollo de esta epidemia mundial de obesidad, creando el llamado "ambiente obesogénico". ${ }^{32}$ Se han propuesto, al menos sobre el papel, iniciativas colectivas que comprometen la participación de los diversos agentes sociales referidos, como las escuelas, las industrias alimentarias, las administraciones públicas o los profesionales sanitarios.

Sin embargo, ¿por qué las acciones promovidas por los programas de prevención han supuesto cambios mínimos en el entorno socioeconómico, mientras que se potencian aquéllas dirigidas a los individuos? Los objetivos para alcanzar los llamados estilos de vida saludables continúan centrados en modificar las conductas personales, tales como lograr un equilibrio energético y un peso normal, mejorar los conocimientos nutricionales o aumentar la actividad física. Sobre estos ámbitos se trabaja desde hace tiempo y, aun así, el éxito ha sido más bien modesto. ${ }^{55}$ Ello se debe, en opinión del autor, a que las acciones propuestas no han tenido en cuenta la compleja naturaleza del comportamiento alimentario ni de la cultura. ¿Por qué se insta a modificar los hábitos alimentarios inadecuados y no se proponen medidas eficaces para cambiar el sistema que es el que, al fin y al cabo, favorece la emergencia de ciertas enfermedades en determinados grupos sociales? Las recomendaciones nutricionales no deben perder de vista, además, que los estudios epidemiológicos señalan para muchos países una mayor prevalencia de la obesidad entre las mujeres y entre la población con menor nivel económico y formativo. ${ }^{56-58}$ Entonces ¿por qué emitir todavía mensajes estandarizados sobre la conveniencia de la actual dieta equilibrada si, por razones estructurales o culturales, mucha gente no puede acceder a ella? Por otro lado, referirse al entorno social cuando se trata de buscar las causas o responsabilidades de ciertos problemas significa no definirlo, tal como se acostumbra hacer desde las instituciones, como una especie de nebulosa abstracta ("ambiente obesogénico") y, en consecuencia, difícilmente abordable, sino aprehenderlo en tanto que organización misma de una sociedad. *

En las sociedades industrializadas, ello significa comprender los condicionantes económicos, culturales y políticos que en relación con el capitalismo de consumo afectan todo y a todos: el acceso a los recursos, las relaciones de género, las formas de entender la enfermedad o los cuidados del cuerpo. ¿Se ha dejado de lanzar al mercado productos alimentarios de dudosa calidad nutricional o se han frenado las prescripciones médicas que incentivan, de forma indiscriminada, el seguimiento de dietas y el consumo de fármacos adelgazantes tras intuir, sin excesiva dificultad, su relación con la aparición o aumento de ciertas patologías alimentarias? Si se acepta, por otro lado, que los hábitos alimentarios de algunos grupos sociales han empeorado, es decir, que desde un punto de vista nutricional comen mal y deben aprender a hacerlo bien, la razón de ello debería buscarse en los cambios registrados en la estructura social y la organización económica de estas sociedades. Porque son éstas las que impiden (a través de la discriminación social, las largas jornadas laborales y formativas, la falta de tiempo para organizar la comida familiar, la pérdida generacional de competencias culinarias o la promoción del consumo compulsivo), otros modos de vida más saludables.

\section{Discusión}

Los constructos acerca de la dieta y la regulación del peso corporal no sólo ofrecen información valiosa sobre la naturaleza y los posibles efectos de unas prácticas médicas centradas en pretender cambiar los desordenados estilos de vida a los que han llevado el progreso y

\footnotetext{
* Gracia M, Contreras J. Corps gros, corps malades? Une perspective Socioculturelle. Corps Revue Interdisc 2007:2. En prensa.
} 
la civilización, sino también acerca de cómo sirven para producir y mantener patrones de promoción de la salud; éstos, a pesar de la diversidad cultural y económica de las poblaciones, son muy similares a nivel mundial. Una de las paradojas del proceso de medicalización alimentaria en las sociedades industrializadas es que si bien la divulgación de la dieta equilibrada ha penetrado en el entramado social e, incluso, ha dotado de sentido a los discursos profanos sobre lo que se considera comer bien, no ha logrado modificar sus prácticas alimentarias. Así lo demostraría el incremento de enfermedades crónicas vinculadas con la obesidad. Esta divergencia entre el conocimiento normativo y las prácticas alimentarias cuestiona, en buena medida, la eficacia del actual modelo de prevención e intervención nutricional, en particular por dos razones. En primer lugar porque apenas se ha comenzado a trabajar sobre los factores estructurales que al parecer explicarían el aumento de la prevalencia de esta enfermedad. Es el caso, por ejemplo, de la desigualdad social, la calidad nutricional de algunos productos alimentarios o la promoción del consumo indiscriminado. Por otro lado, los tratamientos para abordarla se adaptan poco a las particularidades biopsicosociales de las personas obesas y se dirigen, sobre todo, a modificar los denominados "estilos de vida" a través del seguimiento disciplinado de dietas y ejercicio físico. Esta visión prescinde del hecho de que la salud, dado que es una motivación importante del consumo alimentario, es sólo uno de los múltiples condicionantes de la alimentación cotidiana y deja de lado que comer mal o bien reviste significados distintos, contradictorios o complementarios según sea que el determinante sea el placer, el bolsillo, la comensalidad, la conveniencia o la enfermedad. Asimismo, este modelo medicalizado basa sus preceptos en dotar de competencias dietéticas y responsabilizar a los individuos de su estado de salud sin comprender, o cuando menos afrontar, que la vida cotidiana de la ciudadanía es heterogénea per se y está articulada por actividades diversificadas y acontecimientos irregulares a menudo incompatibles con la rutina requerida por los apremios dietéticos.

\section{Referencias}

I. Contreras J, Gracia M.Alimentación y cultura. Perspectivas antropológicas. Barcelona: Ariel, 2005.

2. Piddocke S. El sistema del potlatch de los kwakiult del sur: una nueva perspectiva. En: Llobera JR, ed.Antropología económica. Barecelona: Anagrama, 1981.

3. Tannahill R. Food in history. Londres: Penguin Books, 1988. 4. Powdermaker H.An anthropological approach to the problem of obesity. In: Counihan C,Van Esterik, P. eds. Food and culture. A reader. Londres: Routledge, 1997: 370-383.

5.Vigarello G. Lo sano y lo malsano. Madrid:Abadía Lectores, 2006.
6. Mead M. Dietary patterns and food habits. I Am Diet Ass 1943; I9(I):I-5.

7. Soares MC. Agonia da fome. Salvador Bahia: EDUFBA, 2003.

8. Mintz S. Tasting food, tasting freedom: excursions into eating, culture and the past. Boston: Beacon Press, 1996.

9. FAO. El estado de inseguridad alimentaria en el mundo 2006. Disponible en: http://www.fao.org.

10. Gracia M. No comerás. Narrativas sobre la comida, el cuerpo y el género en el Nuevo Milenio. Barcelona: Icaria, 2007. En prensa.

II. Fieldhouse P. Food \& nutrition: customs and culture. Londres:

Chapman \& Hall, 1996.

12. Bell R. Holy Anorexia, Chicago: University of Chicago Press, 1985.

13. Hepworth J.The social construction of anorexia nervosa. Londres: Sage Publications, 1999.

14. Contreras J.Antropología de la alimentación. Madrid: Eudema, 1992.

I5. Sobal J.The medicalization and demedicalization of Obesity. En Maurer D, Sobal J, eds: Eating agendas. Food and nutrition as Social Problems. Nueva York: Aldine de Gruyter, 1995.

16. Manzini I. L'alimentation et la médecine dans le monde antique. En: Flandrin JL, Montanari M. Histoire de l'alimentation. París: Fayard, 1996. 17. Poulain JP. Manger aujourd'hui. Attitudes, normes et pratiques. Toulouse: Privat, 2002

18.Vigarello G. Lo sano y la malsano. Madrid:Abadía Lectores, 2006. 19. Cheyne G. The english malady. Londres: Strachan, 1733.

20. Levenstein HA. Diététique contre gastronomie: traditions culinaires, sainteté et santé dans les modèles de vie américains. En : Flandrin JL, Montanari M. Histoire de l'alimentation. París: Fayard, 1996.

21. Mennell S.All manners of food. Eating and taste in England and France from de Middle Age to the present. Oxford: Basil Blackwell Ltd, 1985.

22. Elias N. El proceso de la civilización. Madrid: Fondo de Cultura Económica, 1989.

23. Turner BS. The discourse of diet. In: Featherstone M, Hepworth M,

Turner B, eds. The body. Social process and cultural theory. Londres: Sage Publications, 1999.

24. Turner BS. The Government of the body: Medical regimens and the rationalization of diet. $\mathrm{Br}$ J Sociol, 1982;33(2):254-269.

25. Levenstein HA. Diététique cotre gastronomie: traditions culinaires, sainteté et santé dans les modèles de vie américains. En: Flandrin JL, Montanari M. Histoire de l'alimentation. París: Fayard, 1996.

26. Conveney J. Food, morals and meaning. Londres: Routledge, 2006.

27. Dapcich V. Guía de la alimentación saludable. Madrid: Sociedad

Española de Nutrición Comunitaria, 2004.

28. Aranceta J (coord). Guía práctica sobre hábitos de alimentación y salud. Madrid: Instituto Omega 3, SENC, 2002.

29. Generalitat de Catalunya. Guia l'alimentació saludable a l'etapa escolar. Barcelona: Generalitat de catalunya, 2005.

30. Ascher F. Le mangeur hypermodern. París: Odile Jacob, 2005.

31. Poulain JP. Les dimesions sociales de l'obesité. En: Obésité, dépistage et prévention chez l'enfant.Expertise collective. París: INSERM, 2000. 32. Ministerio de Sanidad y Consumo. Estrategia para la Nutrición, Actividad Física y Prevención de la Obesidad (NAOS). Madrid: MSC, 2005. 33. Basdevant A, Guy-Gran B. Médicine de l'obésité. París: Flammarion, 2004.

34. Organización Mundial de la Salud. $57^{\mathrm{a}}$ Asamblea Mundial de la Salud. Estrategia Mundial sobre régimen alimentario, actividad física y salud, 2004. 35. Basdevant A, Guy-Gran B. Médicine de l'obésité. París: Flammarion, 2004.

36. Sobal J, Stunkard AJ. Socioeconomic satuts and obesity: a review of the literature. Psychol Bull 1990;105:260-275.

37. Nestlé M. Food politics. Los Angeles: University of California Press, 2002.

38. Campos P.The obesity myth:Why america's obession with weigth is hazardous for your health. Nueva York: Gotham Books, 2004.

39. Maddox GL, Liederman V. Overweight as a social disability with medical implications.J Med Educ 1969;44:2/4-220. 
40. De Labarre M. Dietética y reflexividad: el "cuidado de sí mismo" contemporáneo. En: Millán A, comp.Arbitrario cultural. Racionalidad e irracionalidad del comportamiento comensal. Huesca: La Val de Onsera, 2004 4I. Crawfford R. You are dangerous to your health, the ideology of victim blaming. Inter J Health Serv 1977;7(4):663-680.

42. Ryan W. Blaming the victim. Nueva York:Vintage Books, 1977.

43. Noticia publicada en: http://www.elpais.com (consultada 27/02/2007).

44. Gard M,Wright J. The obesity epidemic. Science, morality and ideology. Londres: Routledge, 2006

45. Ascher F. Le mangeur hypermodern. París: Odile Jacob, 2005.

46. Barona JLL. Salud, enfermedad y muerte. La sociedad valenciana entre

1833 y 1939. Valencia: Institució Alfons El Magnànim, 2002.

47. Tannahill R. Food in history. Londres: Penguin Books, 1988

48. Gracia M.Thoughts on eating risk and its acceptability. The case of transgenic foods (AGMs). Braz J Nutrit 2004; I7(2): I25-I49.

49. Peretti-Watel P. Sociologie du risque. París:Armand Colin, 2000

50. Douglas M,Wildavsky A. Risk and culture. California: University of California Press, 1983.
5I. Boltanski L, Thévenot L. De la justification: les économies de la grandeur. Paris: Gallimard, I99I.

52. Lupton D. Food, risk and subjectivity. In:Williams SJ, ed. Health, medicine and society, key theories, futur agendas. Londres: Routledge, 2000.

53. Menéndez LE. El malestar actual de la antropología o de la casi imposibilidad de pensar lo ideológico. Rev Antropol Soc 2002; I 1:39-87. 54. Parra-Cabrera S. Modelos alternativos para el análisis epidemiológico de la obesidad como problema de salud pública. Rev Saude Publica 1999;33:3|4-325.

55. Farré R.Alimentación y nutrición contemporáneas: realidad y futuro. En: Salas-Salvadó J. La alimentación y la nutrición a través de la historia. Barcelona: Glosa, 2005.

56. Sobal J. Commentary: globalization and the epidemiology of obesity. Inter J Epidemiol 200 I;30: I I36- I I37.

57. Aranceta-Bartrina J. Prevalencia de obesidad en España. Med Clin (Bar) 2005; I 25(I2):460-466.

58. Barquera S. Sobrepeso y obesidad. México: Instituto de Salud Pública, 2006 